\title{
Relativistic quantum chaos in Robertson-Walker cosmologies
}

\author{
Roman Tomaschitz \\ Service de Physique Théorique de Saclay, F-91191 Gif-sur-Yvette, France \\ and Instituts Internationaux de Physique et de Chimie Solvay, Université Libre de Bruxelles, \\ C.P. 231-Campus Plaine, Bd. du Triomphe, B-1050 Brussels, Belgium
}

(Received 25 February 1991; accepted for publication 17 April 1991)

\begin{abstract}
Open Robertson-Walker cosmologies of multiple spatial connectivity provide a challenging example for the possible influence of the global topological structure of space-time on the laws of microscopic motion. Free geodesic motion is investigated in such cosmologies in the context of first quantization. A unique localized wave field, a solution of the Klein-Gordon equation, is found as a consequence of the topological structure of the spacelike slices $t=$ const of the manifold. This solution is closely related to the collection of the bounded chaotic trajectories. The link is provided by the quasi-self-similar limit set of the group of covering transformations on the boundary of the universal covering space of the spacelike sections. It is this fractal set from which the covering geodesics of the bounded trajectories emerge, its Hausdorff measure and dimension determine the localized wave field.
\end{abstract}

\section{INTRODUCTION}

We consider Robertson-Walker (RW) cosmologies whose spacelike slices $t=$ const. are three-manifolds of multiple connectivity, infinite volume, and negative sectional curvature. A typical class of such manifolds is that of smooth thickened surfaces: imagine a two-sphere with some handles attached and imagine the material of which this surface is formed as thick. One gets a three-dimensional space with an interior and exterior boundary, topologically $I \times S$, fibering over a finite interval $I$, the fibers $S$ being compact Riemann surfaces of genus $g \geqslant 2$.

If the boundaries are removed such three-manifolds can be endowed with a metric of constant sectional curvature, that becomes singular at the boundary and gives rise to infinite volume.

The global topological structure of the $\mathrm{RW}$ cosmologies we treat here is thus $\mathbb{R}^{(+)} \times I \times S$, where $\mathbb{R}^{(+)}$is the real (positive) axis. The construction and the geometrical and topological properties of such manifolds will be dealt with in Sec. II.

Geodesic motion in $\mathbb{R}^{(+)} \times I \times S$, which is locally endowed with a RW line element, we will treat (Sec. III) by embedding the manifold into its universal covering space $\mathbb{R}^{(+)} \times B^{3}$. Here, $B^{3}$ denotes hyperbolic space, a shell of the Minkowski hyperboloid, or the Poincaré ball, isometric to it. We will find that there is a special class of trajectories in $\mathbb{R}^{(+)} \times I \times S$, namely those that stay during the whole time evolution in a finite domain that is expanding at the same rate as three-space itself.

These bounded trajectories have covering geodesics in $\mathbb{R}^{(+)} \times B^{3}$ whose initial or end points lie in the limit set of the group of covering transformations. This limit set constitutes a fractal quasi-self-similar curve on the boundary of $B^{3}$. The bounded trajectories are, as in the nonrelativistic case, chaotic, ${ }^{1}$ having the Bernoulli property.

On the other hand, with the limit set of the covering group there is also associated a localized, square-integrable wave field, a solution of the Klein-Gordon equation on $\mathbb{R}^{(+)} \times I \times S$. In Sec. IV we give an integral representation of the space part of this wave field in terms of the Hausdorff measure of the limit set, which in the classical case determines the bounded trajectories.

This localized state as well as the existence of bounded chaotic trajectories is an effect of the global topological structure of the space, and is absent in the traditional open RW models of simple spatial connectivity.

In Secs. IV and V we discuss the time evolution of the energy of wave fields, especially in the early and late stages of the cosmic expansion, and in Sec. V we give some examples, the static case, de Sitter space, and finally a space that is flat, with an expansion factor that is linear in time.

\section{RW GEOMETRIES OF MULTIPLE CONNECTIVITY AND NEGATIVE SPATIAL CURVATURE}

Cosmological line elements complying with the principles of homogeneity and isotropy can be represented as ${ }^{2-4}$

$$
d s^{2}=-c^{2} d t^{2}+a^{2}(t) d \sigma^{2},
$$

where $d \sigma$ is the line element of a three-dimensional space of constant curvature, which we will assume to be negative. The expansion factor $a(t)$ determines the Gaussian curvature of the spacelike slices $t=$ const.; cf. (5) and (6). The metric $d \sigma^{2}$ is usually represented as

$$
d \sigma^{2}=R^{2}\left[d \rho^{2}+\sinh ^{2}(\rho)\left(d \vartheta^{2}+\sin ^{2}(\vartheta) d \varphi^{2}\right)\right],
$$

$0 \leqslant \rho \leqslant \infty$, and has sectional Gaussian curvature $-1 / R^{2}$.

Introducing a new time variable

$$
t^{\prime}(t)=\int_{\text {const. }}^{t} \frac{d t}{a(t)}
$$

we can make (1) conformal to a static metric

$$
d s^{2}=\tilde{a}^{2}\left(t^{\prime}\right)\left[-c^{2} d t^{\prime 2}+d \sigma^{2}\right],
$$

$\tilde{a}\left(t^{\prime}\right):=a\left(t\left(t^{\prime}\right)\right)$.

In the following we use a form of (2) that is conformal to the line element of Euclidean three-space, the change of coordinates 


$$
\sinh \rho=\frac{2 r / R}{1-r^{2} / R^{2}}, \quad 0 \leqslant r / R \leqslant 1,
$$

transforms $a^{2}(t) d \sigma^{2}$ of (2) in the metric of the Poincaré ball $B^{3}, r:=|\mathbf{x}|<R$, cf. Refs. 5 and 6,

$$
a^{2}(t) d \sigma^{2}=\frac{4 a^{2}(t) d \mathbf{x}^{2}}{\left(1-|\mathbf{x}|^{2} / R^{2}\right)^{2}}
$$

In $B^{3}$ we will construct representations of the threemanifolds that form the spacelike sections of our models.

In the discussion of the wave equation in Sec. IV we need explicit formulae for the curvature scalar of the line elements (1) and (3). Using the sign convention for the curvature tensor as in Refs. 3 and 7, the curvature scalar $\widehat{R}_{\text {space }}$ of the sections $t=$ const. of (1), (3) reads

$$
\hat{R}_{\text {space }}=6 K=-6 / a^{2}(t) R^{2},
$$

where $a(t)$ is dimensionless and $K$ the Gaussian curvature of the two-sections of the spacelike slices.

The four-dimensional curvature scalar $\widehat{R}$ of metric (1) or (3) then reads ${ }^{7,8}$

$$
\begin{aligned}
\hat{R} & =6\left[\frac{-1}{a^{2}(t) R^{2}}+\frac{\ddot{a}(t)}{c^{2} a(t)}+\frac{\dot{a}^{2}(t)}{c^{2} a^{2}(t)}\right] \\
& =6\left[\frac{-1}{R^{2} \tilde{a}^{2}\left(t^{\prime}\right)}+\frac{\tilde{a}\left(t^{\prime}\right)}{c^{2} \tilde{a}^{3}\left(t^{\prime}\right)}\right]
\end{aligned}
$$

the dots denote derivatives with respect to the time variables. If $a(t)$ is a constant, then $\widehat{R}$ reduces to $\widehat{R}_{\text {space }}$. In Sec. V we will encounter an example with $R=0, \quad \widehat{R}_{\text {space }} \neq 0$ $(a(t)=\Lambda t)$.

As in Refs. 1, 9, and 10 we model three-space $I \times S$ in the Poincaré ball $B^{3}$ as a non-Euclidean polyhedron with face identification (analogous to the modeling of a torus of zero curvature in the Euclidean plane by identifying the sides of a square; see also Ref. 11 for Riemann surfaces). We emphasize that this polyhedron does not change in time, nor does the radius $R$ of the Poincaré ball, if we use the metric (1), (4) on $I \times B^{3}$. What varies in $B^{3}$ is the Gaussian curvature (5) and the geodesic distance between two points, measured via (4).

For the sake of self-containedness we sketch shortly the construction of polyhedra in $B^{3}$ that give rise to manifolds $I \times S$.

The faces of the polyhedron lie on totally geodesic planes, i.e., on spherical caps orthogonal to the boundary sphere $S_{\infty}$ of $B^{3}$, where the metric (4) gets singular. Geodesics in $B^{3}$ are arcs of circles orthogonal to $S_{\infty}$.

The identification of the faces is carried out by elements of the invariance group $\mathrm{SL}(2, \mathbb{C})$ of the metric (4), for explicit formulae for the group action in $B^{3}$ see Refs. 1 and 6 . The polyhedron has two open ends, namely faces lying on $S_{\infty}$, that are not identified, and whicis constitute the two boundary components of $I \times S$.

Due to these open ends the volume of the polyhedron measured by (4) is infinite, $\mathbb{R}^{(+)} \times I \times S$ belongs therefore to the open models, the spacelike fibers have infinite volume, and $B^{3}$ is their universal covering manifold. ${ }^{12,13}$ The group
$\Gamma$ of covering transformations is generated by the face-pairing transformations of the polyhedron. This group, applied to the polyhedron, say $F$, gives a tesselation $\Gamma(F)$ of the covering space $B^{3}$ that gets filled with isometric images of $F$, which cover $B^{3}$ completely without overlappings. There are accumulation points of images of $F$, located on $S_{\infty}$, constituting a closed fractal Jordan curve $\Lambda(\Gamma)$ on $S_{\infty}$, the limit set of $\Gamma$.

Figures 1(a)-1(d) show tesselations of $S_{\infty}$, which are induced from the tiling of $B^{3}$, and stereographically projected onto the complex plane. The tiling of the second connection component, enclosing the point at infinity, has not been drawn. The large black polygon is an open end of the polyhedron $F$, one of the boundary surfaces of $I \times S$, if its sides (circular arcs) are properly identified in pairs. The tesselations are obtained by applying $\Gamma$ to this polygon. The genus of the fibers $S$ is 19 in all four examples; in fact the polygons have many more sides that are sitting on the vertices and are many orders of 10 smaller than the visible ones. There are bounds on the Hausdorff dimensions of such limit sets that can be circumvented only by increasing the genus of the fibers. ${ }^{14}$

Likewise the second boundary surface of $I \times S$ is a polygon containing the point at infinity, $\Gamma$ applied to it tesselates the remaining part of $\mathbb{C}$ and provides the exterior approximation to $\Lambda(\Gamma)$.

From the tesselation one can read off the Hausdorff dimension $\delta$ of $\Lambda(\Gamma)$. It is related to the number of tiles that one needs to get a uniformly accurate approximation ${ }^{15,16}$ of the curve by the tiling: $\delta(\mathrm{a})=1.386, \delta(\mathrm{b})=1.393$, $\delta(\mathrm{c})=1.381, \delta(\mathrm{d})=1.452$ (all up to \pm 0.003 , for the calculation of $\delta$ see Ref. 1 ).

The topological structure does not determine the global metric structure of three-space nor of the four-manifold, locally determined by (1). In fact the four examples in Figs. 1(a) -1 (d) correspond to nonisometric manifolds $I \times S$ (there does not exist an isotopic distance-preserving diffeomorphism). Only polyhedra whose covering groups $\Gamma$ are $\mathrm{SL}(2, \mathbb{C})$-conjugated are globally isometric.

Though the topological structure does not determine the metric structure, it restricts it however: the space of metrics that are locally of the form (4), and that can be carried by a manifold $I \times S$, can be parametrized by $12(g-1)$ independent real parameters, $g$ the genus of $S$ (deformation space, cf. Refs. 17 and 18). The same holds true for $\mathbb{R}^{(+)} \times I \times S$ and the line element (1), with a given $a(t)$.

The Jordan curve $\Lambda(\Gamma)$ of the limit points of the covering group $\Gamma$ of three-space is crucial in determining the bounded geodesics on the four-manifold. This and the concept of boundedness in a time-dependent metric will be discussed in Sec. III.

Correspondingly, with this curve, its Hausdorff measure, and Hausdorff dimension there is associated a unique localized, square-integrable solution of the Klein-Gordon equation, which has otherwise purely continuous spectrum. The existence of bounded trajectories and localized states is a consequence of the global topological structure, the multiple connectivity of the manifold. This localization phenomenon will be dealt with in Sec. IV. 

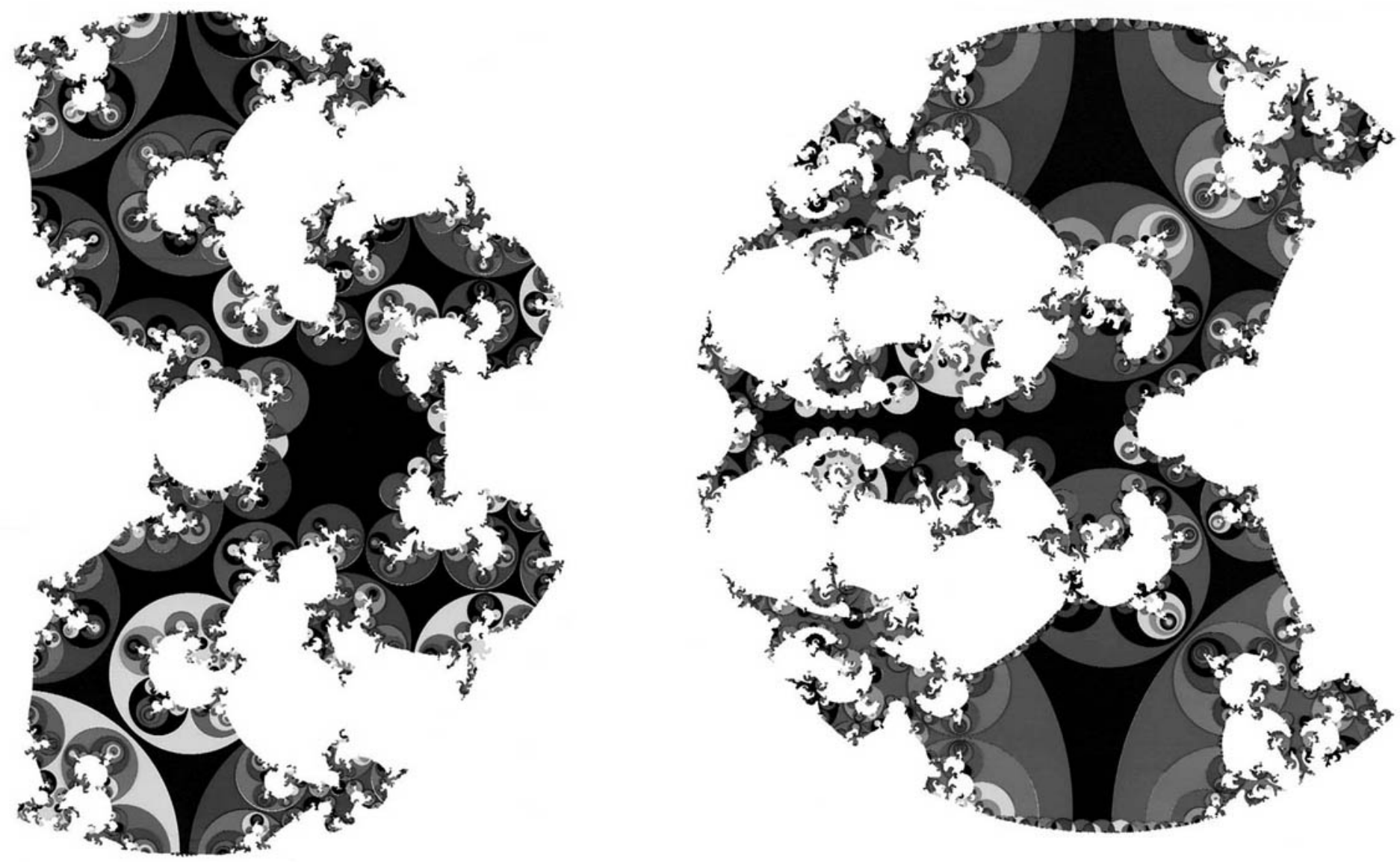

(a)

(c)
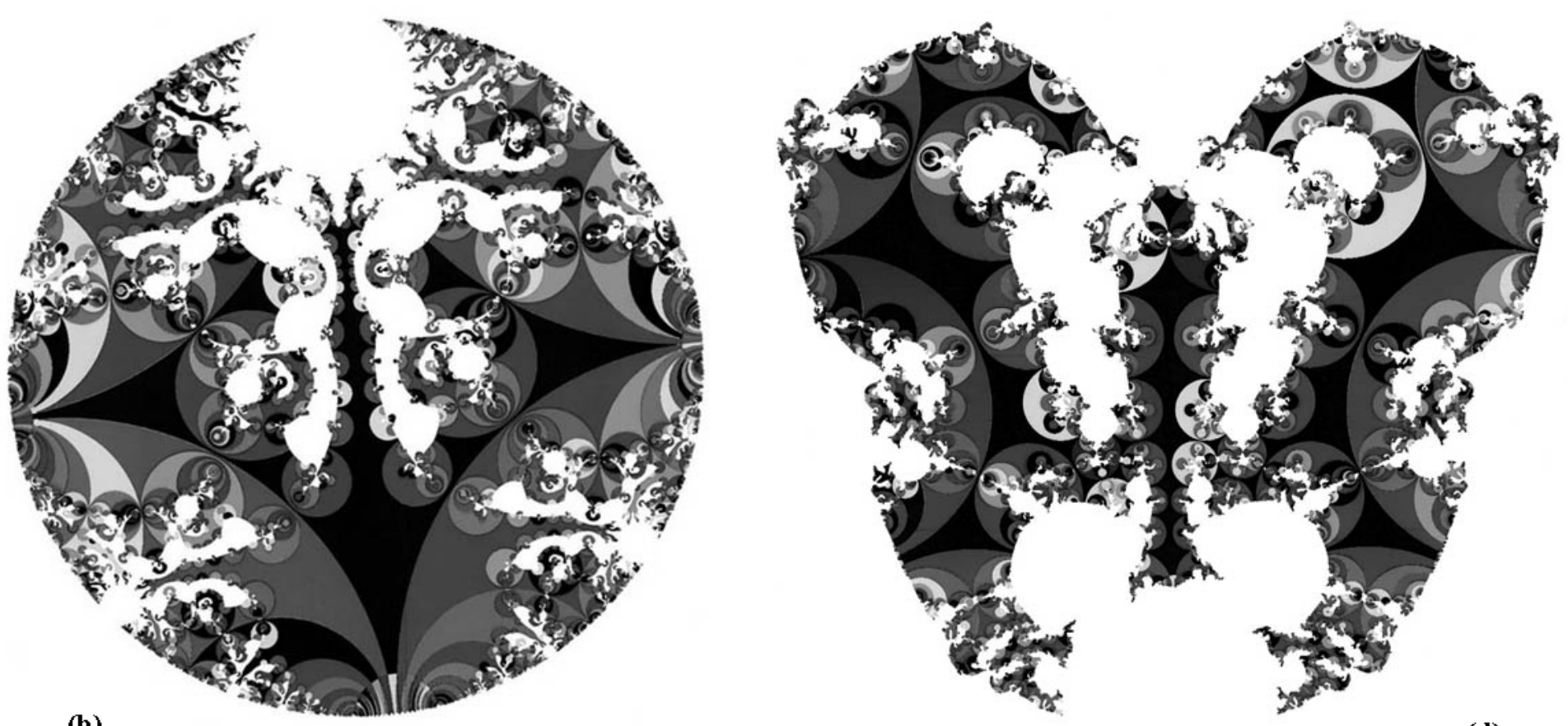

(d)

FIG. 1. (a)-(d) Tilings induced on the boundary $S_{\infty}$ of the universal covering space $B^{3}$ of the spacelike slices $I \times S, t=$ const., by the polyhedral tesselation of $B^{3}$ (cf. Secs. II and III). The images are stereographic projections of $S_{\infty}$, the sphere at infinity of the Poincare ball $B^{3}$, onto the complex plane. Only the component of the tiling on the inside of the quasi-self-similar ${ }^{21}$ Jordan curve $\Lambda(\Gamma)$, which is homeomorphic to a circle, has been drawn. The large black domain, say $f$, is one of the boundary surfaces of three-space $I \times S$. The tiling of $S_{\infty}$ is performed generation by generation by applying the covering group $\Gamma$ to $f$. The tiles are the boundary components of isometric images of $I \times S$, which accumulate at $\Lambda(\Gamma)$. From the limit set $\Lambda(\Gamma)$ the covering geodesics of the bounded trajectories emanate, and its Hausdorff measure and dimension $\delta$ determine a unique localized wave field of the wave equation on the fourdimensional manifold $\mathbf{R}^{(+)} \times I \times S$. The tilings (a)-(d) correspond to nonisometric manifolds of the same topological structure $I \times S, I$ a finite open interval, $S$ a Riemann surface, $g(S)=19 ; \delta(\mathrm{a})=1.386, \delta(\mathrm{b})=1.393, \delta(\mathrm{c})=1.381, \delta(\mathrm{d})=1.452$. 
pp. 257477 in attached file 
approach their rest value, their difference gets of the order $O\left(a^{-2}(t)\right)$.

Nevertheless there is a qualitative difference in the time behavior of the energy of a localized state and that of an unbounded state in the early phase of the expansion. In the case of a square-integrable wave function, cf. (32), the main part of the density $|\psi|^{2}$ stays away from the boundary of three-space (the open ends of $F$ on $S_{\infty}$, cf. Sec. II), and for $a(t) \rightarrow 0|\psi|^{2}$ gets concentrated, for the distances between interior points of the spacelike slices $t=$ const. go to zero. The price to pay for that is localization energy. On the other hand, if $a(t) \rightarrow \infty$ the distances between interior points increase to infinity, $\psi$ gets delocalized, resembling more and more an unbounded state.

\section{A universe that is Minkowskian with the topology of} $\mathbb{R}^{+} \times I \times S$

If we take in (1) the linear expansion factor $a(t)=\Lambda t$, $R=c / \Lambda$ in $(4), 0 \leqslant t \leqslant \infty$, the curvature scalar in (6) vanishes. Indeed, changing coordinates in (1), (4), cf. Ref. 2,

$$
r^{\prime}=2 c t \frac{r / R}{1-r^{2} / R^{2}}, \quad t^{\prime}=t \frac{1+r^{2} / R^{2}}{1-r^{2} / R^{2}},
$$

we get the Minkowski line element in polar coordinates. But in these coordinates space and time get mixed up in the boundary conditions, and the wave equation is not separable.

Defining $v$ as in (53), we get as a normalized fundamental system of Eq. (29) with $\lambda=\Lambda^{2} c^{-2} \delta(2-\delta)$ as in the previous example

$$
\varphi(t)=(\pi / 2)^{1 / 2} e^{-i \pi v / 2} \Lambda^{-3 / 2} t^{-1} H_{v}^{(2)}\left(m c^{2} t / \hbar\right), \text { (59) }
$$

and its complex conjugate. $\left(H_{v}^{(2)}\right.$ is a Hankel function, ${ }^{30}$ the phase factor is needed if $v^{2}<0$.)

The asymptotic expansion of (59) to the order we need is

$$
\begin{aligned}
\varphi(t)= & e^{i \pi / 4}\left(\hbar / m c^{2}\right)^{1 / 2}(\Lambda t)^{-3 / 2} e^{-i m c^{2} t / \hbar} \\
& \times\left[1+i B(v) / t+C(v) / t^{-2}+O\left(t^{-3}\right)\right],
\end{aligned}
$$

with

$$
\begin{aligned}
& B(v)=-\frac{1}{2} \frac{\hbar}{m c^{2}}\left(v^{2}-\frac{1}{4}\right), \\
& C(v)=-\frac{1}{8}\left(\frac{\hbar}{m c^{2}}\right)^{2}\left(v^{2}-\frac{1}{4}\right)\left(v^{2}-\frac{9}{4}\right) .
\end{aligned}
$$

For $t \rightarrow \infty$ we have with (44), (60)

$$
E(t, v)=m c^{2}+O\left(t^{-2}\right),
$$

and

$$
\begin{aligned}
& E(t, v)-E(t, v=0) \\
& =-\frac{1}{4}\left(\hbar^{2} / m c^{2}\right) t^{-2} v^{2}\left(v^{2}+15\right)+O\left(t^{-4}\right) .
\end{aligned}
$$

For $t \rightarrow 0$ we have the same qualitative behavior of $\varphi$ and $E$ as in de Sitter space $[a(t)=\Lambda t \approx \sinh (\Lambda t)]$. If $v>0$, $E(t, v) \sim(1 / 2 \pi)(1+v) 2^{2 v} \Gamma^{2}(v) m c^{2}\left(m c^{2} t / \hbar\right)^{-2 v-1}$, e.g., $E\left(t, v=\frac{1}{2}\right) \sim 3 / 2 m c^{2}\left(m c^{2} t / \hbar\right)^{-2}$, and for the continuous spectrum $\operatorname{Im}(v)>0$

$$
\begin{gathered}
E(t, v) \sim t^{-1}\left[c_{1}+c_{2} \cos \left(|v| \log m c^{2} t / \hbar\right)\right. \\
\left.+c_{3} \sin \left(|v| \log m c^{2} t / \hbar\right)\right] .
\end{gathered}
$$

All that has been stated about the de Sitter example applies here too, with obvious modifications. So the parameter $\xi$ in (25) does not enter here at all, because $\widehat{R}=0$, and the exponential decay in (57) is replaced by the algebraic one in (62), as it is in $a^{-2}(t)$.

In the case of $m=0$ we have for the positive frequency solution of (29)

$$
\varphi(t)=\Lambda^{-1 / 2} \kappa^{-1 / 2}(\Lambda t)^{-1} e^{-i \kappa \log \Lambda t},
$$

with $\kappa=[\delta(2-\delta)-1]^{1 / 2}>0$. For $E(t)$ we have from (63)

$$
E(t)=\hbar t^{-1}\left(\kappa+\kappa^{-1}\right)
$$

and $E(t) a(t)=$ const., compare the red shift relation at the end of Sec. III. Since $\kappa$ must be positive that $\varphi$ has the correct time behavior for $t \rightarrow \infty, \delta$ cannot lie in the interval [1,2 [, which excludes a localized wave solution in the case of zero rest mass in this universe, compare our discussion of de Sitter space. The energy asymptotics of models whose expansion factors have power law behavior in the asympototic regions is discussed in Refs. 9 and 10.

\section{CONCLUSION}

Open RW cosmologies of multiple spatial connectivity show localization phenomena foreign to the simply connected open standard models of cosmology.

In the case of classical geodesic motion they manifest themselves in the appearance of bounded chaotic trajectories, in quantum mechanics as localized wave fields. These phenomena are purely topological; the fact that a trajectory is bounded or not does not depend on its energy (there is no threshold value as in Hamiltonian mechanics), but is decided in the universal covering space of the manifold. If its covering trajectories emerge from the limit set of the covering group it is bounded and chaotic, otherwise not.

If the metric is time dependent we no longer have a simple relation between the spectrum of the wave equation and the energy of the corresponding quantum states. Nevertheless there is a qualitative difference in the time evolution of the energy of a localized state and that of an unbounded one, during the early stages of the expansion of the universe (see the discussion of de Sitter space), but also there does not exist something like a threshold value.

On the other hand, the Hausdorff measure of the limit set $\Lambda(\Gamma)$ on the boundary of the universal covering space determines the spatial part of the localized wave field. The Hausdorff dimension of $\Lambda(\Gamma)$ determines the time asymptotics of the energy of the field at the beginning of the expansion. Thus this fractal, quasi-self-similar set provides without any (semi-classical) approximation the link between the collection of the bounded chaotic trajectories and the localized wave field on the manifold.

\section{ACKNOWLEDGMENTS}

I would like to thank Daniel Bessis for initiating my stay in Saclay, and Pierre Verpeaux for crucial help with the graphics.

\footnotetext{
${ }^{1}$ R. Tomaschitz, Physica D 34, 42 (1989).

${ }^{2}$ L. Infeld and A. Schild, A New Approach to Kinematic Cosmology, Phys. Rev. 68, 250 (1945).
} 
${ }^{3}$ L. D. Landau and E. M. Lifshitz, The Classical Theory of Fields (Pergamon, London, 1971).

${ }^{4}$ G. C. McVittie, General Relativity and Cosmology (Chapman and Hall, London, 1956).

${ }^{5}$ L. V. Ahlfors, Mobius Transformations in Several Dimensions, Lecture Notes (Univ. Minnesota, Minneapolis, 1981).

${ }^{6}$ A. F. Beardon, The Geometry of Discrete Groups, Graduate Texts in Math. Vol. 91 (Springer-Verlag, Berlin, 1983).

${ }^{7}$ C. W. Misner, K. S. Thorne, and J. A. Wheeler, Gravitation (Freeman, New York, 1973).

${ }^{8}$ S. Weinberg, Gravitation and Cosmology (Wiley, New York, 1972).

${ }^{9} \mathrm{R}$. Tomaschitz, in Proceedings of a NATO Advanced Research Workshop on Quantum Chaos, held 28 May-1 June 1991 in Copenhagen, edited by P. Cvitanovic (NATO ASI Series, Plenum, New York, to appear).

${ }^{10} \mathrm{R}$. Tomaschitz, in Proceedings of a Conference on Chaotic Dynamics, held 11-20 July 1991 in Patras, Greece, edited by T. Bountis, (NATO ASI Series, Plenum, New York, to appear).

${ }^{11}$ J. Stillwell, Classical Topology and Combinatorial Group Theory, Graduate Texts in Math (Springer Verlag, New York, 1980), Vol. 72.

${ }^{12}$ A. Marden, in Discrete Groups and Automorphic Functions, edited by W. J. Harvey (Academic, London, 1977), pp. 259-293.

${ }^{13}$ I. M. Singer and J. A. Thorpe, Lecture Notes on Elementary Topology and Geometry, Undergraduate Texts in Math (Springer-Verlag, New York, 1976).

${ }^{14} \mathrm{R}$. Tomaschitz (in preparation).

${ }^{15}$ P. D. Lax and R. S. Phillips, J. Funct. Anal. 46, 280 (1982).

${ }^{16}$ R. S. Phillips and P. Sarnak, Acta Math. 155, 173 (1985).
${ }^{17}$ L. Bers, Ann. Math. 91,570 (1970).

${ }^{18}$ S. L. Krushkal, B. N. Apanasov, and N. A. Grusevskii, Kleinian Groups and Uniformization in Examples and Problems, Translation of Math. Monographs Vol. 62 (Amer. Math. Soc., Providence RI, 1986).

${ }^{19}$ P. Jordan, Schwerkraft und Weltall (Vieweg und Sohn, Braunschweig, 1955), 2nd ed.

${ }^{20}$ D. Sullivan, Publ. Math. I. H. E. S. 50, 171 (1979).

${ }^{21}$ D. Sullivan, Seminar on Conformal and Hyperbolic Geometry, I. H. E. S. Lecture Notes, 1982 (unpublished).

${ }^{22}$ N. D. Birrell and D. C. W. Davies, Quantum Fields in Curved Space (Cambridge U.P., Cambridge, 1982).

${ }^{23}$ S. W. Hawking and G. F. R. Ellis, The Large Scale Structure of Spacetime (Cambridge U.P., Cambridge, 1973).

${ }^{24}$ J. Elstrodt, F. Grunewald, and J. Mennicke, Russ. Math. Surv. 38, 137 (1983).

${ }^{25}$ S. J. Patterson, Acta Math. 136, 241 (1976).

${ }^{26}$ S. J. Patterson, in Analytical and Geometrical Aspects of Hyperbolic Space, London, Math. Soc. Lecture Notes 111, edited by D. B. A. Epstein (Cambridge U.P., London, 1987), pp. 281-323.

${ }^{27}$ S. Helgason, Harmonic Analysis on Homogeneous Spaces (BirkhauserVerlag, Berlin, 1981).

${ }^{28}$ D. A. Hejhal, The Selberg Trace Formula for PSL $(2, R)$, Lecture Notes in Mathematics Vol. 1001 (Springer-Verlag, Berlin, 1983), Vol. 2.

${ }^{29}$ P. A. Perry, J. reine angew. Math. (Crelle) 398, 67 (1989).

${ }^{30}$ W. Magnus, F. Oberhettinger, and R. P. Soni, Formulas and Theorems for the Special Functions of Mathematical Physics, Grundlehren (Springer-Verlag, Berlin, 1966), Vol. 52. 